\title{
Windows executable software for the progressive demasking task
}

\author{
StÉphane Dufau \\ CNRS and Aix-Marseille University, Marseille, France \\ Michate Stevens \\ Ghent University, Ghent, Belgium \\ AND \\ Jonathan Grainger \\ CNRS and Aix-Marseille University, Marseille, France
}

\begin{abstract}
The progressive demasking (PDM) task is a perceptual identification task using degraded stimulus presentation procedures, but with the advantage of providing response time (RT) measures on each trial. Participants simply press a response button whenever they recognize a word. In this article, we present newly developed, easy-to-use freeware for PDM. Considering the difficulty of programming such a paradigm with standard experimental software, we developed a program based on high-level C libraries to be executed on Windows XP computers. The program execution is millisecond accurate for RT measurements and generates the fewest possible display duration errors.
\end{abstract}

In order to study the cognitive processing involved in printed word perception, researchers manipulate stimulus word characteristics in different experimental tasks (see Haberlandt, 1994, for a review). One popular task is the perceptual identification task, in which stimuli are degraded and participants' errors are recorded (e.g., Havens \& Foote, 1963). This task has been particularly favored in the study of early perceptual processing during word identification. The downside of such a task, however, in comparison with tasks using response time (RT) measurements (e.g., the LDT), is that many trials involve no response (when participants fail to identify the stimulus). Furthermore, the offline, unspeeded responses typically used in such tasks could be influenced by factors intervening after word identification. Nevertheless, perceptual identification tasks have an advantage over the more standard lexical decision and naming tasks that are typically used to study visual word recognition (Balota \& Chumbley, 1984) in that the former require a unique stimulus identification. It should nevertheless be noted that, as Grainger and Jacobs (1996) argued, all tasks can be usefully exploited as long as one has an accurate model of the different processes (e.g., activation and decision) involved in response generation.

The progressive demasking (PDM) task was developed by Grainger and Segui (1990) in order to provide an online perceptual identification task with RT measures on each trial..$^{1}$ In this task, a word stimulus is presented, interspersed with a masking stimulus. Through successive display changes, the duration of the stimulus is increased and the duration of the mask decreased, giving the impression that the word emerges from the mask.

These presentation conditions slow down the recognition process, allowing a more sensitive measure of ongoing perceptual processing. However, presentation conditions can be adjusted in such a way that RTs are in the order of $1 \mathrm{sec}$, hence eliminating slow guessing strategies that likely intervene in offline versions of this task (e.g., the gating task for auditory word recognition-Grosjean, 1980).

Evidence for the increased sensitivity of the PDM task in comparison with that of the more standard LDT was provided by Grainger and Segui (1990) in an experiment manipulating target word frequency and the frequency of the target word's orthographic neighbors. Expressed as percent average RT, the effects of orthographic neighborhood increased from $2.6 \%$ in the LDT to $10.6 \%$ in PDM (and the difference was further exaggerated when only lowfrequency words were considered: $2.6 \%$ vs. $14.6 \%$ ). In a similar vein, the effects of stimulus word frequency were $3.3 \%$ in lexical decision and $8.4 \%$ in PDM. Furthermore, the errors generated in the PDM task revealed interesting patterns, such as the fact that some orthographically similar words of different lengths (and thus not orthographic neighbors according to the classic $\mathrm{N}$ definition- see Coltheart, Davelaar, Jonasson, \& Besner, 1977) were given in response (e.g., the French word votre ["your"] was reported instead of vote ["vote"]). This comparison of lexical decision and PDM certainly highlighted the potential advantages of using PDM in research on visual word recognition. 
Since the work of Grainger and Segui (1990), the PDM task has been used in several other investigations of visual word recognition. Carreiras, Perea, and Grainger (1997) compared performance in the lexical decision task (LDT), the PDM task, the naming task, and a semantic categorization task. They found a strong correlation in mean RT per item across LDT and PDM, and much weaker correlations with either of those tasks and naming and semantic categorization RTs. Indeed, a factor analysis of the data obtained in all four tasks revealed three orthogonal factors: one on which both LDT and PDM loaded most strongly, one on which only naming RTs loaded, and one on which only semantic categorization RTs loaded. The most straightforward interpretation of this analysis is that both LDT and PDM are good reflections of the time required to recognize visually presented words. On the other hand, both the naming and semantic categorization tasks are heavily contaminated by other influences on performance-phonological-articulatory for naming, semantic relatedness for semantic categorizationthat are undeniably of interest in their own right.

Finally, the PDM task has also been used in more specialized investigations of visual word recognition. Dijkstra, Van Heuven, and Grainger (1998; Van Heuven, Dijkstra, \& Grainger, 1998) used the PDM task in their investigations of bilingual word recognition. Ferrand and Grainger (2003) used PDM to study phonological influences on visual word recognition by comparing recognition of heterographic homophones (e.g., maid and made) and nonhomophones. Baayen and Schreuder (2003) used the PDM task in their investigation of the influence of morphological family size on visual word recognition. Finally, Bijeljac-Babic, Millogo, Farioli, and Grainger (2004) tested a variant of the PDM task (the luminance increment paradigm, which involves a gradual increase in stimulus luminance as opposed to stimulus duration) in a study of word recognition in beginning readers. Indeed, the PDM task is a particularly easy task to use with beginning readers, and we expect to see more research applying this technique in investigations of reading acquisition.

Given the utility of PDM as an additional tool for studying visual word recognition (and recognition of other types of alphanumeric stimuli), it is important to have a freely available computer program that accurately executes this task. Unfortunately, no standard experimental software available today (e.g., DMDX [Forster \& Forster, 2003], E-Prime-www .pstnet.com/products/e-prime) provides a straightforward implementation of the PDM task. The main reason for this is the way in which stimulus characteristics are interpreted line by line in such software. A simple word list with standard software leads to a major time discrepancy (hundreds of milliseconds) between the desired and the measured display duration of stimuli. However, a more complex programming style is also usually permitted by such software, thus allowing for a significant improvement in timing accuracy. Nevertheless, in all attempts to implement the PDM task using such software, stimulus timing accuracy was found not to be stable across an entire experiment.

From these attempts and failures, we concluded that the PDM task requires dedicated software. This was the case for the software used in the above-cited research, all using DOS executable Turbo Pascal programs for PCs or C programs for Apple Macintosh computers with special hardware extensions. In the present work, we introduce dedicated software for the PDM task. The program, called PDM, is compiled and executable on a Windows XP computer, and its sources are available on the Internet under a GNU/GPL license. The idea of using dedicated software was prompted by a former Turbo Pascal program that was built in the early 1990 s for the DOS environment. As Myors (1999) noted, programs under DOS were executed without interruptions, meaning that timing accuracy was perfect. Within a multitask environment such as Windows XP, program execution is interrupted from time to time by other programs, possibly leading to timing errors. However, Windows XP allows the setting of program priority, and 2007 computers are sufficiently fast to deal with nearly perfect timing for such a task.

\section{DESCRIPTION OF THE TASK}

The PDM task manipulates the display duration of a masking stimulus such as a string of hash marks (\#\#, considered here as noise) and a target stimulus such as the word TABLE (considered here as signal). On any given trial, the pair [\#\#; TABLE] is displayed consecutively several times. A typical trial takes the form of several cycles, each of which is formed of the successive presentation of the mask followed by the target. These cycles continue until the target is identified by the participant. A response is then made by pressing a designated key on the keyboard. On successive cycles within a given trial, the signal-tonoise ratio is increased. At the beginning of a trial (i.e., on the first cycle), the target stimulus duration is typically set at a minimal value close to zero, and the mask duration is set at some upper limit (e.g., $200 \mathrm{msec}$ ). As the cycles follow each other, the mask duration decreases and the target duration increases in the same proportion. Table 1 shows the display duration (in milliseconds) of cycles in a trial for the pair [\#\#\#; TABLE].

\section{PDM SOFTWARE}

In developing the PDM software, we aimed to fulfill the following specifications: (1) the program needs to be compiled, for faster execution; (2) the program should be executable within a Windows environment; (3) the program execution should be millisecond accurate for RT measure-

Table 1

Ihustration of Typical Timing (in Milliseconds) for Mask and Target Stimuli in the PDM Task

\begin{tabular}{cccc}
\hline Cycle & $\begin{array}{c}\text { Mask Duration } \\
\text { (e.g., }\end{array}$ & $\begin{array}{c}\text { Target Duration } \\
\text { (e.g., TABLE) }\end{array}$ & $\begin{array}{c}\text { Total } \\
\text { Duration }\end{array}$ \\
\hline 1 & 195 & 15 & 210 \\
2 & 180 & 30 & 210 \\
3 & 165 & 45 & 210 \\
$\vdots$ & $\vdots$ & $\vdots$ & $\vdots$ \\
12 & 30 & 180 & 210 \\
13 & 15 & 195 & 210 \\
14 & 0 & until response & until response \\
\hline
\end{tabular}


ments and generate the least possible display errors; and (4) the program should be easily configured by users via stimuli and configuration files. In this section, we describe how the new user can start using PDM software.

To become familiar with the software, one can first execute the program (called pdm.exe) via a launcher called PDM_run.bat using its default configuration. In its default configuration, the program is set up to run five PDM trials in a window on the desktop. Before the start of the trials, the program will prompt for the participant number and stimulus file number. The choice of the participant number is free; the stimulus file number should be 1 , since only one example stimulus file is supplied with the program. When the program has read the stimulus file (located in the "user_input" directory), it will prompt the user to press the space bar to start the experiment. When the user does so, the first word-mask pair is displayed progressively until the space bar is pressed again. The program then requests that the word that has been recognized be typed using the computer keyboard. The word should be typed and the return key pressed to begin the next trial. Two pauses are prompted during this first execution. To resume the experiment after a pause, the return key should be pressed. After the last trial, an output file is written in the "user_output" directory. Its name is data_SnFm.txt, where $n$ is the participant number and $m$ is the stimulus file number. The .txt file contains information about each trial, such as stimulus identity, participant's response, RT, and extra information related to timing accuracy. More detailed information about the output file can be found in the software documentation. In the remainder of this article, we describe the contents of the stimulus and configuration files and the inner workings of the program, and provide some information concerning timing accuracy.

\section{User-Defined Text Files}

The PDM software package contains several files, including an example stimulus file and a configuration file. These are plain text files that can be easily manipulated using any text editor.

Stimulus file. The stimulus file holds the mask and target identities in a two-column-like arrangement. Masks are set in the first column and targets in the second. The program uses the mask and target written on one line per trial. The stimulus file is called stim $N$.txt, where $N$ is the number of the file. Several files can be prepared with different random orderings of the stimuli. At start-up, the program will ask which file number to read.

Configuration file. The other parameters for the experiment can be set in the configuration file (called pdm .ini). The first set of parameters controls the screen and timing parameters, such as refresh rate and program priority. For each parameter, all possible values and the default value are listed.

The second set of parameters controls the duration of the different events in a trial. With the third set of parameters, the instructions-such as "press the space bar" at the beginning of the first trial-can be altered to fit the language context of the experiment. The last set of parameters controls the fonts used for words and masks. Three different fonts (Arial, Courier, and Times) can be used in four modes (normal, bold, italics, and bold italics) and 16 different sizes (ranging from 8 to 72 points). If necessary, user-supplied fonts can be used by the program. To do that, Windows font files have to be converted to font files readable by PDM. The process of generating such font files is described in the documentation.

\section{Binary Files}

The PDM program is written in the C programming language. It relies on the TScope library (Stevens, Lammertyn, Verbruggen, \& Vandierendonck, 2006) for displaying the stimuli and registering responses with millisecond accuracy. The program runs on Windows 2000/XP computers and is available as an archive containing a precompiled executable and all necessary DLLs or font files. ${ }^{2}$ The archive also contains the source files of the program.

\section{Timing Considerations}

Because it is a precompiled, binary executable, the PDM program should execute rapidly and accurately in most cases. However, since Windows XP is a multitasking environment, some timing errors might occur when the PDM program is run. There are two possible timing errors: stimulus display duration errors and RTEs. In order to minimize display errors, we implemented a specific algorithm that relies on the vertical synchronization signal. For example, when one needs a display that stays on for five display cycles, the algorithm does not count five vertical synchronization signals but only starts waiting for a vertical synchronization signal a few milliseconds before the expected appearance of the fifth one. Therefore, missing one of the vertical synchronization signals early in a stimulus display interval does not increase the display duration of that stimulus. Only if the last synchronization signal is missed will the stimulus be displayed longer than expected. When a timing error occurs during PDM execution, it is reported in the output data of each trial in two error measures called stimulus timing error (STE) and response timing error (RTE). STEs occur when the target or mask stays on the screen longer than expected after the PDM program misses the vertical synchronization signal. The STE reports such errors as the number of screen cycles added to the display duration. RTEs occur when the interval between two successive scans of the keyboard is longer than $1 \mathrm{msec}^{3}$

\section{Validation of the Stimulus Timing Algorithm}

To assess the quality of the display timing and error detection algorithm, an extra test mode was added to the program. When the PDM program is run in this mode, a trigger is sent to the parallel port at the onset of the first mask of each trial. Masks are presented in white on a black text background; targets are presented in black on a white text background. After the presentation of all mask/target cycles in a trial, the program jumps to the next trial even if no response was registered. Because of its noninteractive nature, this mode can be used to easily obtain a software estimate of the display timing performance of every computer on which an experimenter intends to run PDM. 
All display timing errors encountered will be logged in the data files. The trigger sent to the parallel port at the start of each trial and the alternations between black mask fields and white target fields make a further hardware test possible. In our test setup, we ran the PDM program for a series of trials on a Dell Optiplex GX 520 PC (Intel Pentium D $3 \mathrm{GHz}, 1 \mathrm{~GB}$ of RAM and an onboard graphics card) with a Fijutsi Siemens 151E 15-in. CRT screen attached to it. ${ }^{4}$ Every millisecond, a Fluke 196C oscilloscope sampled the status of the parallel port of the computer and of an optocoupler attached to the center of the screen. These samples were then relayed to a second computer. On this computer, a National Instruments Labview 8.2 program was used to deduce the length of each mask and stimulus field of each trial from the raw samples. On a CRT screen, the image is drawn by activating small phosphor elements on the screen surface with an electron beam. If activated, the phosphor element lights up for about $2 \mathrm{msec}$. It then remains black until the next screen refresh cycle. The length of the (white) stimulus fields can therefore be deduced by counting the number of peaks in the optocoupler's signal. The length of the (black) mask fields can be deduced from the time interval between series of peaks in the optocoupler's signal.

In a first test run, in which PDM was run in highpriority mode and with no other applications running, not a single STE occurred over a series of 1,000 trials that had 26 stimulus-mask alternations each. Both the software and the hardware agreed that each field had exactly the desired length. In a second run, we intentionally ran PDM using a very bad setup in which STEs were very likely to occur. An infinite loop program was running on the computer and caused a 40\%-50\% processor load. PDM was run with normal priority and gave up its allocated time slice at the start of every stimulus field, allowing the other process to run freely. In this run, 123 of the 26,000 stimulus fields stayed on screen too long. Importantly, there was perfect agreement between the error estimates generated by PDM and by the hardware setup-exactly the same fields were reported as having an STE. Of the 123 errors, there was only one case in which the error was larger than one cycle.

Two conclusions can be drawn from this hardware test. First, when run properly on a well-maintained computer, STEs are very unlikely to occur. Second, if STEs do occur, they will be detected accurately and logged in the data file. Therefore, users are encouraged to do a test run on each computer before proceeding with experimental runs. This will accurately inform them of whether a specific computer is up to the task or not. If errors are encountered, the experimenter can tune the display and timing parameters in the pdm.ini file, modify the Windows environment, or, as a last resort, test the PDM program on another computer. All possible manipulations are described in the documentation.

\section{CONCLUSIONS}

The PDM task is a technique for degrading visual stimulus presentation in order to provide an online measure of stimulus identification time. In the introduction to this work, we summarized some of the potential advantages of the PDM task as a technique for studying visual word recognition. Unlike popular alternative tasks used to study visual word recognition, PDM requires explicit identification of the stimulus, and unlike traditional perceptual identification techniques, PDM provides an online measure of RT.

We believe that the task has been underexploited until now, mostly because it is difficult to implement using current software packages. Therefore, the present work was designed to facilitate the use of this paradigm by providing user-friendly, freely accessible software for PDM. In this work, we have described a program based on high-level C libraries to be executed on Windows XP computers. This program provides a simple and accurate means of using the PDM task in an experiment.

Future research could use the PDM task to study visual word recognition not only in skilled readers, but also during reading acquisition and in patients with reading impairments. Because of its simplicity, the task is particularly suited for research with beginning readers. Finally, we aim to provide a version of PDM developed for use with any type of visual stimulus, such as objects and faces, thus further expanding the perspectives for the application of this task in cognitive research.

\section{AUTHOR NOTE}

We thank Phil Allen for his useful comments on an earlier version of this work. Thanks to Juan Segui, the inventor of PDM and a continual source of inspiration for researchers in our field. Thanks also to Pascal Mestdagh for setting up the hardware test rig that was used in the validation study. Correspondence concerning this article should be addressed to S. Dufau, Laboratoire de Psychologie Cognitive, Université de Provence, Centre Saint Charles, Case D, 3 place Victor Hugo, 13331 Marseille CEDEX 03 (e-mail: stephane.dufau@univ-provence.fr).

\section{REFERENCES}

Allen, P. A., Smith, A. F., Lien, M. C., GrabBe, J., \& MuRPhy, M. D. (2005). Evidence for an activation locus of the word-frequency effect in lexical decision. Journal of Experimental Psychology: Human Perception \& Performance, 31, 713-721.

BAAYEN, R. H., \& SChreUder, R. (2003). Morphological structure in language processing. Berlin: Mouton de Gruyter.

Balota, D. A., \& Chumbley, J. I. (1984). Are lexical decisions a good measure of lexical access? The role of word frequency in the neglected decision stage. Journal of Experimental Psychology: Human Perception \& Performance, 10, 340-357.

Bijeluac-Babic, R., Millogo, V., Farioli, F., \& Grainger, J. (2004). A developmental investigation of word length effects in reading using a new on-line word identification paradigm. Reading \& Writing, 17, 411-431.

Carreiras, M., Perea, M., \& Grainger, J. (1997). Effects of orthographic neighborhood in visual word recognition: Cross-task comparisons. Journal of Experimental Psychology: Learning, Memory, \& Cognition, 23, 857-871.

Coltheart, M., Davelaar, E., Jonasson, J. T., \& Besner, D. (1977). Access to the internal lexicon. In S. Dornic (Ed.), Attention and performance $V I$ (pp. 535-555). Hillsdale, NJ: Erlbaum.

Dijkstra, T., VAn Heuven, W. J. B., \& Grainger, J. (1998). Simulating cross-language competition with the bilingual interactive activation model. Psychologica Belgica, 38, 177-196.

Ferrand, L., \& Grainger, J. (2003). Homophone interference effects in visual word recognition. Quarterly Journal of Experimental Poychology, 56A, 403-419.

Feustel, T. C., Shiffrin, R. M., \& Salasoo, A. (1983). Episodic and lexical contributions to the repetition effect in word identification. Journal of Experimental Psychology: General, 112, 309-346. 
Forster, K. I., \& Forster, J. C. (2003). DMDX: A Windows display program with millisecond accuracy. Behavior Research Methods, Instruments, \& Computers, 35, 116-124.

GrAINGER, J., \& JACOBS, A. M. (1996). Orthographic processing in visual word recognition: A multiple read-out model. Psychological Review, 103, 518-565.

Grainger, J., \& Segui, J. (1990). Neighborhood frequency effects in visual word recognition: A comparison of lexical decision and masked identification latencies. Perception \& Psychophysics, 47, 191-198.

GrosJEAN, F. (1980). Spoken word recognition processes and the gating paradigm. Penception \& Psychophysics, 28, 267-283.

HaberLandT, K. (1994). Cognitive psychology. Needham Heights, MA: Allyn \& Bacon.

Havens, L. L., \& Foote, W. E. (1963). The effect of competition on visual duration threshold and its independence of stimulus frequency. Journal of Experimental Psychology, 65, 6-11.

MYoRs, B. (1999). Timing accuracy of PC programs running under DOS and Windows. Behavior Research Methods, Instruments, \& Computers, 31, 322-328.

Plant, R. R., Hammond, N., \& Turner, G. (2004). Self-validating presentation and response timing in cognitive paradigms: How and why? Behavior Research Methods, Instruments, \& Computers, 36, 291-303.

Plant, R. R., Hammond, N., \& Whitehouse, T. (2003). How choice of mouse may affect response timing in psychological studies. Behavior Research Methods, Instruments, \& Computers, 35, 276-284.
SHIMIZU, H. (2002). Measuring keyboard response delays by comparing keyboard and joystick inputs. Behavior Research Methods, Instruments, \& Computers, 34, 250-256.

SteVens, M., Lammertyn, J., Verbruggen, F., \& VANDierenDONCK, A. (2006). TScope: A C library for programming cognitive experiments on the MS Windows platform. Behavior Research Methods, 38, 280-286.

Van Heuven, W. J. B., Dukstra, T., \& Grainger, J. (1998). Orthographic neighborhood effects in bilingual word recognition. Journal of Memory \& Language, 39, 458-483.

\section{NOTES}

1. For similar approaches, see Feustel, Shiffrin, and Salasoo (1983) and Allen, Smith, Lien, Grabbe, and Murphy (2005).

2. Documentation, software, and their associated source files are available under a GNU/GPL license at www.up.univ-mrs.fr/wlpc/pdm.

3. It should be noted that delays that occur within the hardware and microcode of the keyboard cannot be detected by software such as PDM (Plant, Hammond, \& Whitehouse, 2003; Shimizu, 2002).

4. Fast display switches are achieved better using CRT monitors than using TFT panels (Plant, Hammond, \& Turner, 2004).

(Manuscript received January 25, 2007; revision accepted for publication April 11, 2007.) 